\title{
ON ALMOST LOCALLY CONNECTED SPACES
}

\author{
TAKASHI NOIRI
}

(Received March 25, 1982)

Communicated by J. H. Rubinstein

\begin{abstract}
In this paper it is shown that almost local connectedness is hereditary for the subspace that is the union of regular open sets and is preserved under almost-open (in the sense of Singal) $\theta$-continuous surjections.
\end{abstract}

1980 Mathematics subject classification (Amer. Math. Soc.): 54C10, 54D05.

\section{Introduction}

Recently, V. J. Mancuso [3] has introduced and investigated the concept of almost locally connected spaces. In [3], among others, the following theorems have been established:

THEOREM A. Let $f: X \rightarrow Y$ be an almost-open, almost-continuous and connected surjection. If $X$ is almost locally connected and $Y$ is almost-regular, then $Y$ is almost locally connected.

THEOREM B. Let $f: X \rightarrow Y$ be an open, almost-continuous and connected surjection. If $X$ is almost locally connected, then so is $Y$.

The main purpose of the present paper is to improve the previous theorems. In Section 4 it will be shown that almost local connectedness is preserved under almost-open and almost-continuous surjections. By making use of this result we

(c) Copyright Australian Mathematical Society 1983 
shall show in Section 5 that the word "regular open" in Theorem 3.8 of [3] can be replaced by "the union of regular open sets".

\section{Preliminaries}

Throughout this paper spaces mean topological spaces on which no separation axioms are assumed unless explicitly stated. Let $S$ be a subset of a space $X$. The closure of $S$ and the interior of $S$ in $X$ are denoted by $\mathrm{Cl}_{X}(S)$ and $\operatorname{Int}_{X}(S)$ (or simply $\mathrm{Cl}(S)$ and $\operatorname{Int}(S)$ ), respectively. A subset $S$ is said to be regular open (resp. regular closed $)$ if $\operatorname{Int}(\mathrm{Cl}(S))=S(\operatorname{resp} . \mathrm{Cl}(\operatorname{Int}(S))=S)$. The family of all regular open sets of a space $X$ is denoted by $\operatorname{RO}(X)$. A function $f: X \rightarrow Y$ is said to be almost-continuous [12] (resp. $\theta$-continuous [1], weakly-continuous [2]) if for each point $x \in X$ and each open set $V$ of $Y$ containing $f(x)$ there exists an open set $U$ of $X$ containing $x$ such that $f(U) \subset \operatorname{Int}_{Y}\left(\mathrm{Cl}_{Y}(V)\right)$ (resp. $f\left(\mathrm{Cl}_{X}(U)\right) \subset \mathrm{Cl}_{Y}(V)$, $\left.f(U) \subset \mathrm{Cl}_{Y}(V)\right)$.

REMARK 2.1. It is known that continuity $\Rightarrow$ almost-continuity $\Rightarrow \theta$-continuity $\Rightarrow$ weak-continuity and none of these implications is reversible ([6], [12]).

\section{Almost locally connected spaces}

Definition 3.1. A space $X$ is said to be almost locally connected (simply a.l.c.) [3] if for each $x \in X$ and each $G \in \operatorname{RO}(X)$ containing $x$ there exists an open connected set $V$ such that $x \in V \subset G$.

We shall begin by giving a characterization of a.l.c. spaces which will be used in the subsequence.

THEOREM 3.2. The following statements are equivalent for a space $X$ :

(1) $X$ is a.l.c.

(2) The components of regular open sets in $X$ are regular open in $X$.

(3) For each $x \in X$ and each $G \in \operatorname{RO}(X)$ containing $x$, there exists a regular open connected set $V$ such that $x \in V \subset G$.

Proof. (1) $\Rightarrow(2)$ : Let $G \in \operatorname{RO}(X)$ and $C$ be a component of $G$. By Theorem 3.5 of [3], $C$ is open in $X$ and $C \subset \operatorname{Int}_{X}\left(\mathrm{Cl}_{X}(C)\right)$. On the other hand, since $C$ is connected, so is Int ${ }_{X}\left(\mathrm{Cl}_{X}(C)\right)$. Since $C$ is a component of $G, \operatorname{Int}_{X}\left(\mathrm{Cl}_{X}(C)\right) \subset C$. Therefore, we have $C=\operatorname{Int}_{X}\left(\mathrm{Cl}_{X}(C)\right)$ which shows that $C \in \operatorname{RO}(X)$.

$(2) \Rightarrow(3)$ and $(3) \Rightarrow(1)$ are easy and the proofs are thus omitted. 
Definition 3.3. A space $X$ is said to be nearly-compact [11] if every regular open cover of $X$ has a finite subcover.

COROLlARY 3.4. A nearly-compact a.l.c. space has a finite number of components.

Proof. Let $X$ be a nearly-compact a.l.c. space. Since $X \in \mathrm{RO}(X)$, by Theorem 3.2 the family of components of $X$ is a regular open cover of $X$. Therefore, $X$ has a finite number of components.

A space $X$ is said to be weakly-Hausdorff [13] if every point of $X$ is the intersection of regular closed sets.

THEOREM 3.5. A nearly-compact weakly-Hausdorff space $X$ is a.l.c. if and only if every regular open cover of $X$ is refined by a cover consisting of a finite number of regular open connected sets.

Proof. Let $X$ be a nearly-compact a.l.c. space and $\mathscr{V}=\left\{V_{\alpha} \mid \alpha \in \nabla\right\}$ a regular open cover of $X$. By Theorem 3.2, for each $\alpha \in \nabla$ the components $C_{\alpha(j)}$ of $V_{\alpha}$ are regular open in $X$, where $\alpha(j) \in \nabla(\alpha)$. Since $X$ is nearly-compact, there exist a finite subset $\nabla_{0}$ of $\nabla$ and a finite subset $\nabla_{0}(\alpha)$ of $\nabla(\alpha)$ for each $\alpha \in \nabla_{0}$ such that

$$
X=\bigcup\left\{C_{\alpha(j)} \mid \alpha(j) \in \nabla_{0}(\alpha), \alpha \in \nabla_{0}\right\} .
$$

Thus, the family $\left\{C_{\alpha(j)} \mid \alpha(j) \in \nabla_{0}(\alpha), \alpha \in \nabla_{0}\right\}$ is a desirable refinement of $\mathcal{T}$.

Conversely, under the condition that $X$ is a weakly-Hausdorff space and the hypothesis holds, we shall show that $X$ is a.l.c. Let $x \in X$ and $x \in G \in \operatorname{RO}(X)$. Since $X$ is weakly-Hausdorff, for each $y \in X-G$ there exists $U_{y} \in \operatorname{RO}(X)$ such that $y \in U_{y}$ and $x \notin U_{y}$. Then $G \cup\left\{U_{y} \mid y \in X-G\right\}$ is a regular open cover of $X$. By the hypothesis, it has a refinement $\left\{V_{\alpha} \mid \alpha \in \nabla\right\}$ consisting of a finite number of regular open connected sets. There exists an $\alpha_{0} \in \nabla$ such that $x \in V_{\alpha_{0}}$. If $V_{\alpha_{0}} \subset U_{y}$ for some $y \in X-G$, then $x \in U_{y}$. This is a contradiction. Therefore, we obtain $x \in V_{\alpha_{0}} \subset G$. This shows that $X$ is a.l.c.

\section{Preservation theorems}

A function $f: X \rightarrow Y$ is said to be almost-open (simply a.o.R.) [10] if $f(U) \subset$ Int $_{Y}\left(\mathrm{Cl}_{Y}(f(U))\right)$ for every open set $U$ of $X$. A function $f: X \rightarrow Y$ is said to be connected [8] if for each connected set $C$ of $X, f(C)$ is connected in $Y$. The following theorem is an improvement of Theorem $\mathrm{B}$. 
THEOREM 4.1. Let $f: X \rightarrow Y$ be an a.o.R., weakly-continuous and connected surjection. If $X$ is a.l.c., then so is $Y$.

Proof. Let $y \in Y$ and $y \in G \in \operatorname{RO}(Y)$. It follows from Theorem 3.4 of [6] that $f^{-1}(G) \in \operatorname{RO}(X)$. Since $X$ is a.l.c., for $x \in f^{-1}(y)$ there exists an open connected set $U$ of $X$ such that $x \in U \subset f^{-1}(G)$. Since $f$ is a.o.R., we have $f(U) \subset$ $\operatorname{Int}(\mathrm{Cl}(f(U)))$. Since $f$ is connected, $f(U)$ is connected and so is $\operatorname{Int}(\mathrm{Cl}(f(U)))$. Moreover, we obtain

$$
y \in f(U) \subset \operatorname{Int}(\mathrm{Cl}(f(U))) \subset G .
$$

This shows that $Y$ is a.l.c.

We shall show the main theorem of this paper which is an improvement of Theorem A and Theorem B. For this purpose we need some lemmas.

Definition 4.2. A function $f: X \rightarrow Y$ is said to be almost-open (simply a.o.S.) [12] if for each $U \in \mathrm{RO}(X) f(U)$ is open in $Y$.

REMARK 4.3. It is known in [7] that "a.o.S." neither implies "a.o.R.,", nor does "a.o.R." imply "a.o.S.".

LEMMA 4.4. Let $f: X \rightarrow Y$ be a weakly-continuous surjection and $X_{0}$ be a subset of $X$. If $f\left(X_{0}\right)$ is open in $Y$, then the function $f_{0}: X_{0} \rightarrow f\left(X_{0}\right)$, defined by $f_{0}(x)=f(x)$ for each $x \in X_{0}$, is weakly-continuous.

Proof. Put $Y_{0}=f\left(X_{0}\right)$. Let $x \in X_{0}$ and $V_{0}$ be an open set of $Y_{0}$ containing $f_{0}(x)$. Since $Y_{0}$ is open in $Y, V_{0}$ is open in $Y$. By weak-continuity of $f$, there exists an open set $U$ of $X$ containing $x$ such that $f(U) \subset \mathrm{Cl}_{Y}\left(V_{0}\right)$. Put $U_{0}=U \cap X_{0}$, then $U_{0}$ is an open set of $X_{0}$ containing $x$ and $f_{0}\left(U_{0}\right) \subset \mathrm{Cl}_{Y}\left(V_{0}\right) \cap Y_{0}=\mathrm{Cl}_{Y_{0}}\left(V_{0}\right)$. This shows that $f_{0}$ is weakly-continuous.

LeMMA 4.5. Let $f: X \rightarrow Y$ be an a.o.S. weakly-continuous surjection. If $U$ is a regular open connected set of $X$, then $f(U)$ is open connected in $Y$.

Proof. Since $f$ is a.o.S. and $U \in \operatorname{RO}(X), f(U)$ is open in $Y$. It follows from Lemma 4.4 that the function $f_{0}: U \rightarrow f(U)$ is weakly-continuous. Since $U$ is a connected set of $X$, by Theorem 3 of [4] $f_{0}(U)=f(U)$ is connected.

THEOREM 4.6. Let $f: X \rightarrow Y$ be an a.o.S. $\theta$-continuous surjection. If $X$ is a.l.c., then so is $Y$. 
Proof. Let $y \in Y$ and $y \in G \in \operatorname{RO}(Y)$. Every a.o.S. function is weakly-open [7, Lemma 1.4]. Hence, it follows from Theorem 4.4 of [6] that $f^{-1}(G) \in \operatorname{RO}(X)$. Since $X$ is a.l.c., for $x \in f^{-1}(y)$ by Theorem 3.2 there exists a regular open connected set $U$ of $X$ such that $x \in U \subset f^{-1}(G)$. Every $\theta$-continuous function is weakly-continuous. Therefore, by Lemma $4.5 f(U)$ is open connected in $Y$ and $y \in f(U) \subset G$. This shows that $Y$ is a.l.c.

COROLlaRy 4.7. Almost local connectedness is preserved under a.o.S. almost-continuous surjections.

Proof. This is an immediate consequence of Theorem 4.6.

REMARK 4.8. The previous corollary shows that the hypothesis "connected" on $f$ in Theorems A and B and also "almost-regular" on $Y$ in Theorem A can be removed.

In this paper, for simplicity, we call the set $X$ with the topology having $\operatorname{RO}(X)$ as a basis the semi-regularization, denoted by $X_{s}$, of a space $X$.

COROllary 4.9. $A$ space $X$ is a.l.c. if and only if the semi-regularization $X_{s}$ is locally connected.

Proof. Necessity. Let $X$ be a.l.c. The identity function $i_{X}: X \rightarrow X_{s}$ is a.o.S. and continuous. Thus, by Corollary $4.7 X_{s}$ is a.l.c. and it is locally connected by Proposition 3.3 of [3].

Sufficiency. Let $X_{s}$ be locally connected. The identity function $j_{X}: X_{s} \rightarrow X$ is open and almost-continuous. It follows from Corollary 4.7 that $X$ is a.l.c.

THEOREM 4.10. Let $f: X \rightarrow Y$ be an almost-continuous surjection. If $X$ is compact a.l.c. and $Y$ is Hausdorff, then $Y$ is a.l.c.

Proof. Since $f$ is almost-continuous and $X$ is compact, $Y=f(X)$ is nearlycompact [11, Theorem 3.2]. Moreover, since $Y$ is Hausdorff, it is almost-regular [11, Theorem 2.4]. It follows from Theorems 4.9 and 2.5 of [5] that $f_{s}$ is a continuous function of a compact space $X_{s}$ onto a Hausdorff space $Y_{s}$. Therefore, $f_{s}$ is closed. Since $X$ is a.l.c., by Corollary $4.9 X_{s}$ is locally connected and hence $f_{s}\left(X_{s}\right)=Y_{s}$ is locally connected. It follows from Corollary 4.9 that $Y$ is a.l.c. 


\section{Subspaces of a.l.c. spaces}

Theorem 3.8 of [3] states that if $A$ is a regular open set of an a.l.c. space $X$ then the subspace $A$ is a.1.c. The following theorem shows that the hypothesis "regular open" on $A$ in this result can be replaced by "the union of regular open sets".

THEOREM 5.1. If $X$ is an a.l.c. space and $A$ is the union of arbitrarily many regular open sets, then the subspace $A$ is a.l.c.

Proof. It follows from Corollary 4.9 that $X_{s}$ is locally connected. Since the identity function $i_{X}: X \rightarrow X_{s}$ is a.o.S., $i_{X}(A)$ is open in $X_{s}$ and hence the subspace $i_{X}(A)$ is locally connected. The identity function $j_{X}: X_{s} \rightarrow X$ is open and almostcontinuous. Since $A$ is open in $X$, by Lemma 4.4 the induced identity function $\left(j_{X}\right)_{0}: i_{X}(A) \rightarrow A$ is open weakly-continuous. Moreover, every open weakly-continuous function is almost-continuous [12, Theorem 2.3]. Therefore, it follows from Corollary 4.7 that $A$ is a.l.c.

A subset $K$ of a Hausdorff space $X$ is said to be $H$-closed relative to $X$ [9] if for every cover $\left\{\nabla_{\alpha} \mid \alpha \in \nabla\right\}$ of $K$ by open sets of $X$ there exists a finite subset $\nabla_{0}$ of $\nabla$ such that $K \subset \cup\left\{\mathrm{Cl}_{X}\left(V_{\alpha}\right) \mid \alpha \in \nabla\right\}$.

COROLlary 5.2. If a space $X$ is a.l.c. Hausdorff and $K$ is $H$-closed relative to $X$, then $X-K$ is a.l.c.

Proof. Let $x \in X-K$. Since $X$ is Hausdorff, for each $y \in K$ there exist regular open sets $V(y)$ and $W(y)$ containing $x$ and $y$, respectively, such that $V(y) \cap \mathrm{Cl}(W(y))=\varnothing$. Since $\{W(y) \mid y \in K\}$ is a cover of $K$ by open sets of $X$, there exists a finite subset $K_{0}$ of $K$ such that

$$
K \subset \bigcup\left\{\mathrm{Cl}_{X}(W(y)) \mid y \in K_{0}\right\} .
$$

Put $V_{x}=\cap\left\{V(y) \mid y \in K_{0}\right\}$, then $x \in V_{x} \in \operatorname{RO}(X)$ and $V_{x} \subset X-K$. It follows from Theorem 5.1 that $X-K$ is a.l.c.

\section{References}

[1] S. Fomin, 'Extensions of topological spaces', Ann. of Math. 44 (1943), 471-480.

[2] N. Levine, 'A decomposition of continuity in topological spaces', Amer. Math. Monthly 68 (1961), 44-46.

[3] V. J. Mancuso, 'Almost locally connected spaces', J. Austral. Math. Soc. Ser. A 31 (1981), 421-428. 
[4] T. Noiri, 'On weakly continuous mappings', Proc. Amer. Math. Soc. 46 (1974), 120-124.

[5] T. Noiri, 'On $\delta$-continuous functions', J. Korean Math. Soc. 16 (1980), 161-166.

[6] T. Noiri, 'A note on $\eta$-continuous functions', J. Korean Math. Soc. 18 (1981), 37-42.

[7] T. Noiri, 'Semi-continuity and weak-continuity', Czechoslovak. Math. J. 31 (1981), 314-321.

[8] W. J. Pervin and N. Levine, 'Connected mappings of Hausdorff spaces', Proc. Amer. Math. Soc. 9 (1958), 488-495.

[9] J. Porter and J. Thomas, 'On H-closed and minimal Hausdorff spaces', Trans. Amer. Math. Soc. 138 (1969), 159-170.

[10] D. A. Rose, 'Weak continuity and almost continuity', preprint.

[11] M. K. Singal and Asha Mathur, 'On nearly-compact spaces', Boll. Un. Mat. Ital. (4) 2 (1969), $702-710$

[12] M. K. Singal and A. R. Singal, 'Almost-continuous mappings', Yokohama Math. J. 16 (1968), 63-73.

[13] T. Soundararajan, 'Weakly Hausdorff spaces and the cardinality of topological spaces', General topology and its relations to modern analysis and algebra III, Proc. Conf. Kampur, 1968, pp. 301-306 (Academia, Prague, 1971).

\section{Department of Mathematics}

Yatsushiro College of Technology

Yatsushiro-shi, Kumamoto-ken

866 Japan 